\title{
New Bounds from a Search for Muonium to Antimuonium Conversion
}

\author{
L. Willmann ${ }^{1}$, P. V. Schmidt, ${ }^{1}$ H. P. Wirtz, ${ }^{2}$ R. Abela, ${ }^{3}$ V. Baranov, ${ }^{4}$ J. Bagaturia, ${ }^{5}$ W. Bertl, ${ }^{3}$ R. Engfer, ${ }^{2}$ \\ A. Großmann, ${ }^{1}$ V. W. Hughes, ${ }^{6}$ K. Jungmann, ${ }^{1}$ V. Karpuchin, ${ }^{4}$ I. Kisel, ${ }^{4}$ A. Korenchenko, ${ }^{4}$ S. Korenchenko, ${ }^{4}$ \\ N. Kravchuk, ${ }^{4}$ N. Kuchinsky, ${ }_{4}^{4}$ A. Leuschner, ${ }^{2}$ V. Meyer, ${ }^{1}$ J. Merkel, ${ }^{1}$ A. Moiseenko, ${ }^{4}$ D. Mzavia, ${ }^{5}$ G. zu Putlitz, ${ }^{1}$ \\ W. Reichart, ${ }^{2}$ I. Reinhard, ${ }^{1}$ D. Renker,${ }^{3}$ T. Sakhelashvilli, ${ }^{5}$ K. Träger, ${ }^{1}$ and H. K. Walter ${ }^{3}$ \\ ${ }^{1}$ Physikalisches Institut, Universität Heidelberg, D-69120 Heidelberg, Germany \\ ${ }^{2}$ Physik Institut, Universität Zürich, CH-8057 Zürich, Switzerland \\ ${ }^{3}$ Paul Scherrer Institut, CH-5232 Villigen PSI, Switzerland \\ ${ }^{4}$ Joint Institute of Nuclear Research, RU-141980 Dubna, Russia \\ ${ }^{5}$ Tbilisi State University, GUS-380086 Tbilisi, Georgia \\ ${ }^{6}$ Physics Department, Yale University, New Haven, Connecticut 06520-8121
}

(Received 13 July 1998)

\begin{abstract}
A new upper limit for the probability of spontaneous muonium to antimuonium conversion was established at $P_{\mathrm{MM}} \leq 8.3 \times 10^{-11}(90 \%$ C.L.) in $0.1 \mathrm{~T}$ magnetic field, which implies consequences for speculative extensions to the standard model. Coupling parameters in $R$-parity-violating supersymmetry and the mass of a flavor diagonal bileptonic gauge boson can be significantly restricted. A $Z_{8}$ model with radiative mass generation through heavy lepton seed and the minimal version of 331 models are disfavored. [S0031-9007(98)08068-5]
\end{abstract}

PACS numbers: 11.30.Fs, 11.30.Hv, 13.10.+q, 36.10.Dr

At present, all confirmed experimental experience is in agreement with conserved lepton numbers. Several solely empirical laws appear to hold simultaneously. including multiplicative and additive schemes [1]. No associated symmetry has yet been identified, thus leaving lepton numbers in a unique status in physics, since flavor mixing in the quark sector is well established and described by the Cabibbo-Kobayashi-Maskawa matrix. The standard model in particle physics assumes additive lepton family number conservation, and any observed violation would be a clear indication of new physics. In many speculative theories, which extend the standard model in order to explain some of its features such as parity violation in the weak interactions or $\mathrm{CP}$ violation, lepton flavors are not conserved. These theories have motivated a variety of dedicated sensitive searches for rare decay modes of muons and kaons [2] and for neutrino oscillations.

Of particular interest is the muonium atom $(\mathrm{M}=$ $\left.\mu^{+} e^{-}\right)$which consists of two leptons from different generations. As the electromagnetic part of the binding is well described by electroweak standard theory it renders the possibility of a search for additional, yet unrevealed electron-muon interactions. A spontaneous conversion of muonium into antimuonium $\left(\overline{\mathrm{M}}=\mu^{-} e^{+}\right)$would violate the additive lepton family number conservation by two units; however, it is allowed by a multiplicative law. This process could play a decisive role in many speculative models (Fig. 1) [3-9].

The measurements reported here were performed with the muonium-antimuonium conversion spectrometer (MACS) whose design is based on the observation of $\mathrm{M}$ atoms in vacuo. In matter the possible conversion is strongly suppressed mainly due to the loss of symmetry between $M$ and $\bar{M}$ due to the possibility of $\mu^{-}$transfer in collisions involving $\overline{\mathrm{M}}[10,11]$. The required signature of a conversion process is the coincident identification of both the electron and positron released in the decay of the antiatom $[12,13]$. An energetic electron $\left(e^{-}\right)$arises from the decay $\mu^{-} \rightarrow e^{-}+\nu_{\mu}+\bar{\nu}_{e}$ with a characteristic Michel energy distribution extending to $53 \mathrm{MeV}$ [14], and a positron $\left(e^{+}\right)$appears with an average kinetic energy of $13.5 \mathrm{eV}$ corresponding to its momentum distribution in the atomic $1 s$ state of $\overline{\mathrm{M}}$ [15].

The setup has a large acceptance for these charged final state particles (Fig. 2). Its symmetry for detecting $M$ and $\bar{M}$ decays through reversing all electric and magnetic fields is exploited in regular measurements of the $\mathrm{M}$ atom production yield which is required for normalization and, in addition, for monitoring detector performance. As a particular advantage, systematic uncertainties arising from corrections for efficiencies and acceptances of various detector components cancel out.

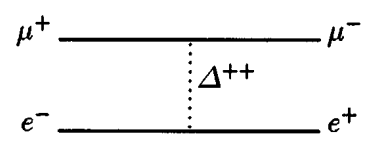

(a)

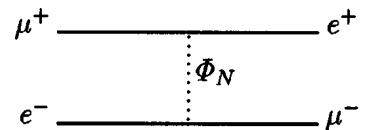

(c)
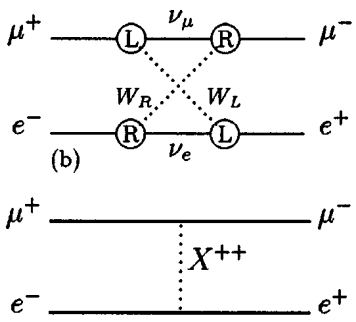

(d)
FIG. 1. Muonium-antimuonium conversion in theories beyond the standard model. The interaction could be mediated, e.g., by (a) doubly charged Higgs boson $\Delta^{++}[3,4]$, (b) heavy Majorana neutrinos [3], (c) a neutral scalar $\Phi_{N}$ [5], e.g., a supersymmetric $\tau$-sneutrino $\tilde{\nu}_{\tau}[6,7]$, or (d) a bileptonic flavor diagonal gauge boson $X^{++}[8,9]$. 


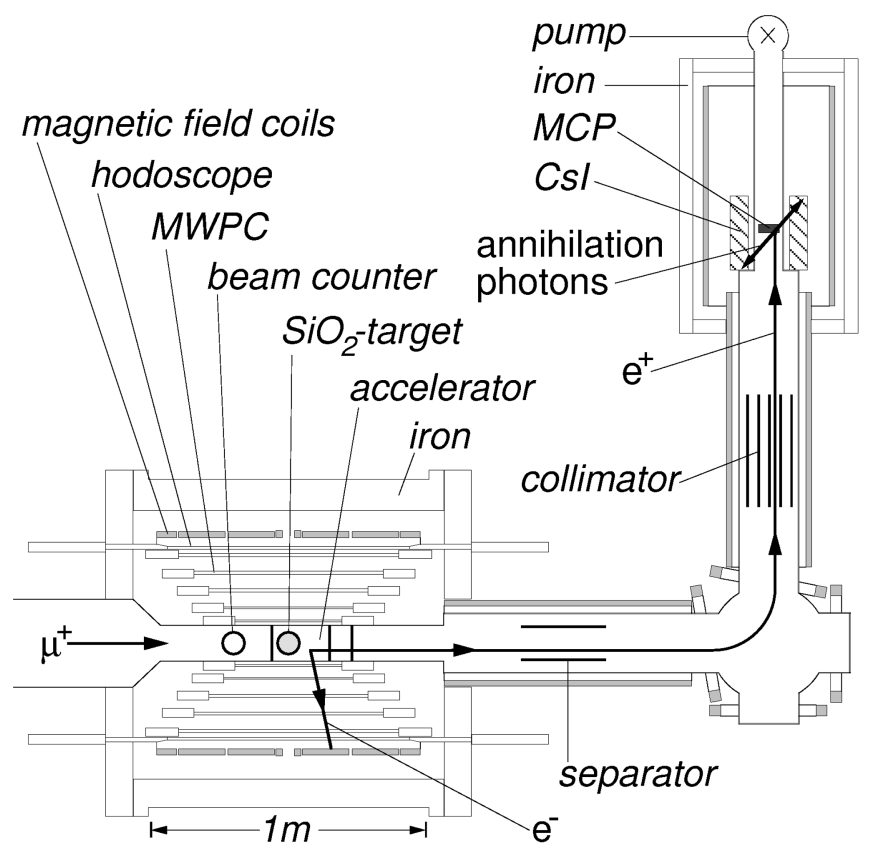

FIG. 2. The MACS apparatus at PSI searching for muoniumantimuonium conversion. The signature requests the energetic $e^{-}$from the $\mu^{-}$decay of $\overline{\mathrm{M}}$ in a magnetic spectrometer in coincidence with the atomic shell $e^{+}$, which is accelerated and magnetically guided onto a microchannel plate (MCP), and at least one annihilation photon in a CsI calorimeter.

The experiment utilizes the world's brightest continuous surface muon channel $\pi$ E5 [16] at the Paul Scherrer Institut (PSI) in Villigen, Switzerland. It provides a central momentum $p=26 \mathrm{MeV} / c$, a momentum bite $\Delta p / p=5 \%$, and rates up to $8 \times 10^{6} \mu^{+} / \mathrm{s}$. The beam passes through a $280 \mu \mathrm{m}$ scintillation counter and a $270 \mu \mathrm{m}$ Mylar degrader. Muonium atoms are formed by electron capture with 61(3)\% efficiency after stopping the $\mu^{+}$in a $\mathrm{SiO}_{2}$ powder target of thickness $8 \mathrm{mg} / \mathrm{cm}^{2}$ and supported in vacuo by a $25 \mu \mathrm{m}$ aluminum foil with $30^{\circ}$ inclination with respect to the muon beam axis. Most of the atoms emerge from the powder grains into the intergranular voids. Then, on average, $3.3 \%$ of them leave the target surface with thermal Maxwell-Boltzmann velocity distribution at $300 \mathrm{~K}$ [17].

When searching for $\overline{\mathrm{M}}$ decays the energetic $e^{-}$is detected in a magnetic spectrometer operated at $B_{0}=$ $0.1 \mathrm{~T}$ magnetic field and covering $0.73 \times 4 \pi$ solid angle around the $\mathrm{M}$ production target. It has five concentric multiwire proportional chambers with radii of 8.2 to $32.0 \mathrm{~cm}$ and active lengths of 38 to $80 \mathrm{~cm}$. They are all equipped with two planes of segmented helical cathode stripes for measuring radial, angular, and axial coordinates. The momentum resolution at $50 \mathrm{MeV} / c$ is $54(2) \%$, yielding a probability of $10^{-5}$ for misidentifying the charge of the particle. It is limited by the $2 \mathrm{~mm}$ wire spacing. The chambers are surrounded by a 64 -fold segmented hodoscope. Subsequent to the $\mu^{-}$decay the atomic shell $e^{+}$is accelerated to typically $7 \mathrm{keV}$ in a two stage electrostatic device. It is guided in an axial $0.1 \mathrm{~T}$ magnetic field along a $3 \mathrm{~m}$ long transport region onto a microchannel plate (MCP) detector with resistive anode readout. A $35 \mathrm{mg} / \mathrm{cm}^{2}$ magnesium oxide coated carbon foil in front of this device provides secondary electrons and hence yields a 4-fold enhancement of the detectors efficiency to 64(2)\%. Furthermore, it reduces background counts of low energy ions trapped in the magnetic field [18]. The transport system is momentum selective due to a $90^{\circ}$ horizontal bend of radius $35 \mathrm{~cm}$ and a collimator consisting of $40 \mathrm{~cm}$ long, $1 \mathrm{~mm}$ thick, and $9 \mathrm{~mm}$ separated copper sheets which act to suppress particles with longitudinal momenta exceeding $750 \mathrm{keV} / c$ because their gyration radii exceed $4.5 \mathrm{~mm}$ in the magnetic guiding field. The field gradient in the bend region causes a vertical drift for charged particles proportional to their momenta. It is compensated for $7 \mathrm{keV} e^{+}$by a transverse electrostatic field region preceding the bend which also deflects low energy $\mu^{+}$and ions.

Positrons are uniquely identified by annihilation radiation when striking the MCP which is centered inside a barrel-shaped 12-fold segmented pure CsI crystal detector. This detector had 4.5(3) ns time and 350(20) keV energy resolution (FWHM). Positrons were required to deposit an energy between $E_{\gamma}=0.2$ and $1.0 \mathrm{MeV}$ and to be detected within $\left|t_{\text {CsI }}\right|<6$ ns of a hit on the MCP. Using $e^{+}$from radioactive sources the acceptance for at least one of two $511 \mathrm{keV}$ annihilation photons was determined to be $\varepsilon_{\mathrm{CsI}, 1 \gamma}=79(4 \%)$ for all measurements.

The transport path has $80(2) \%$ transmission and conserves transverse spatial information of the decay vertex. It can be reconstructed radially to $8.0(4) \mathrm{mm}$ and axially to $8.6(5) \mathrm{mm}$ (FWHM) if, in addition, track parameters are used from the energetic $e^{-}$. The limitations on the position resolution arise at high energies again from proportional chamber wire spacing and at low energies from multiple scattering in the $1 \mathrm{~mm}$ carbon fiber beam tube.

During data taking, every $5 \mathrm{~h}$ the $\mathrm{M}$ production yield was determined at low beam rates $\left(2 \times 10^{4} \mu^{+} / \mathrm{s}\right)$ using a method which is based on a model established in preceding experiments $[13,17]$. The number of atoms in the fiducial volume was determined mainly from the distribution of time intervals $t_{\text {decay }}$ between a beam counter signal from the incoming muon and the detection of the atomic electron on the MCP (Fig. 3). On average, $5.0(2) \times 10^{-3}$ of the incoming $\mu^{+}$were observed to decay as $\mathrm{M}$ atoms in vacuo. The $\mathrm{SiO}_{2}$ targets were replaced twice a week, since the $\mathrm{M}$ yield deteriorated on a time scale of a week associated with the release of $\mathrm{H}_{2} \mathrm{O}$ molecules from the powder.

The final search result was obtained in three data-taking periods with a total duration of six months distributed over four years (Table I) during which the sensitivity of the instrument was constantly improved. In total $N_{M}=5.6(1) \times 10^{10} \mathrm{M}$ atoms in vacuo were investigated for $\bar{M}$ decays. Two major sources of potential background were identified: (i) accidental coincidences of energetic $e^{-}$produced by Bhabha scattering of $e^{+}$from M 

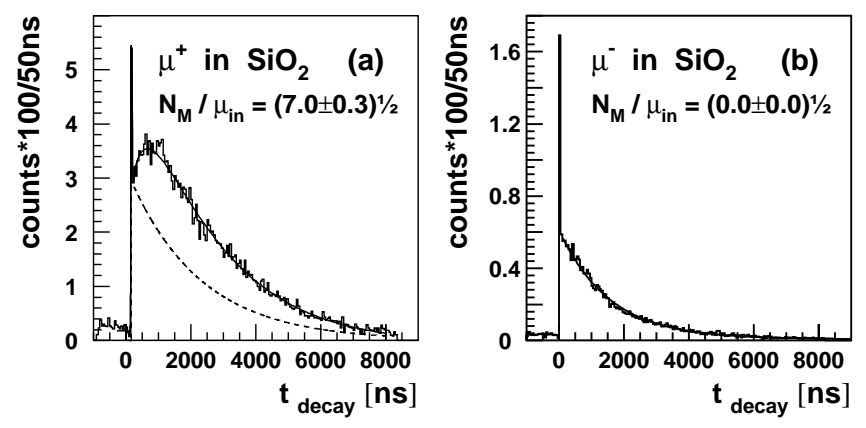

FIG. 3. Muonium production was continually monitored using the characteristic time distribution $t_{\text {decay }}$ of atomic electrons on the MCP (a). The indicated exponentially decaying background was verified by demonstrating that there is only such background for negative muons on $\mathrm{SiO}_{2}$ powder (b).

decays and scattered $e^{+}$on the MCP and (ii) the allowed muon decay mode $\mu \rightarrow e^{+} e^{+} e^{-} \nu_{e} \bar{\nu}_{\mu}$ with branching ratio $3.4 \times 10^{-5}$, which could release an energetic $e^{-}$, and low energy $e^{+}$, which are detected, and the second $e^{+}$escapes unobserved. The observed real $e^{+}$rate was of order $2 \mathrm{~s}^{-1}$. The $e^{+}$time of flight (TOF) is the time difference between corresponding events in the hodoscope and the MCP. Its expected value was determined in $\mathrm{M}$ decays to 76(1) ns with a 3.3(1) ns wide (FWHM) distribution. A narrow coincidence window of $\pm 4.5 \mathrm{~ns}$ was applied to suppress both background processes. Transverse momenta $p_{T}$ of energetic $e^{-}$were accepted between 15 and $90 \mathrm{MeV} / c$, which cuts $50(3) \%$ of the Bhabha scattering events but only 8(2)\% of the Michel spectrum contents. For the rare muon decay the bend of the $e^{+}$transport system spoils the vertex reconstruction due to the significantly higher $e^{+}$momenta. The decay vertex had to be reconstructed within $12 \mathrm{~mm}$ radially and was required to be not more than $3 \mathrm{~cm}$ upstream of the $\mathrm{SiO}_{2}$ target.

There was one event from the final data acquisition period which passed all of these required criteria within three standard deviations of each of the relevant distributions which were established in the regular $\mathrm{M}$ control measurements prior to processing the search data (Fig. 4). The expected background due to accidental coincidences is 1.7(2) events and was evaluated using data with a $\mathrm{TOF}-\mathrm{TOF}_{\text {expected }}>4.5 \mathrm{~ns}$.

Assuming Poissonian statistics with the known background an upper limit on the conversion probability at
90\% C.L. can be found from the sum of all cycles

$$
P_{\mathrm{MM}}(0 \mathrm{~T})<\frac{3.0\left(1+1.5 \sigma_{r}^{2}\right)}{N_{\mathrm{M}} \varepsilon_{\mathrm{CsI}, 1 \gamma} S_{B}\left(B_{0}\right) S_{\mathrm{vol}}} .
$$

Here $\sigma_{r}^{2}$ is the sum of the squares of the parameters' relative uncertainties [19]. The finite fiducial volume of diameter $9 \mathrm{~cm}$ and length $10 \mathrm{~cm}$ and the finite observation time are taken into account by a correction factor $S_{\mathrm{vol}}$, which was determined in a Monte Carlo simulation of the conversion process and which can be averaged over different data acquisition periods using weighting with $N_{\mathrm{M}}$. We find as the combined result $P_{\mathrm{MM}}(0 \mathrm{~T}) \leq 8.3 \times 10^{-11} / S_{B}\left(B_{0}\right)$ (details of individual cycles are listed in Table I). The factor $S_{B}\left(B_{0}\right)$ describes the suppression of the conversion in the external magnetic field $B_{0}$ due to the removal of degeneracy between corresponding levels in $\mathrm{M}$ and $\overline{\mathrm{M}}$. It depends on the interaction type (Table II). The reduction is strongest for $(V \pm A) \times(V \pm A)[20,21]$. For these cases the traditionally quoted upper limit for the coupling constant is $G_{\mathrm{MM}}=G_{\mathrm{F}} \sqrt{P_{\mathrm{MM}}(0 \mathrm{~T}) / 2.56 \times 10^{-5}} \leq 3.0 \times 10^{-3} G_{\mathrm{F}}$ ( $90 \%$ C.L.), where $G_{\mathrm{F}}$ is the Fermi coupling constant.

This new result, which exceeds previous bounds [13] for $P_{\mathrm{MM}}(0 \mathrm{~T})$ by a factor of 35 , has some impact on speculative models. A proposed $Z_{8}$ model is ruled out with more than four generations of particles where masses could be generated radiatively with heavy lepton seeding [5].

A new lower limit of $m_{X^{ \pm \pm}} \geq\left(2.6 \mathrm{TeV} / c^{2}\right) g_{3 l}(95 \%$ C.L.) on the masses of flavor diagonal bileptonic gauge bosons is found in grand unified theory (GUT) models, which is well beyond the value extracted from direct searches, measurements of the muon magnetic anomaly, or high energy Bhabha scattering $[8,22]$, with $g_{3 l}$ of order unity and depending on the details of the underlying symmetry. For 331 models our result translates into $m_{X^{ \pm \pm}} \geq 850 \mathrm{GeV} / c^{2}$, which disfavors their minimal version in which an upper bound of $800 \mathrm{GeV} / c^{2}$ has been extracted from electroweak parameters $[9,23]$. The 331 model is viable in an extended form with, e.g., a Higgs octet [24].

In the framework of $R$-parity-violating supersymmetry $[6,7]$, the bound on the coupling parameters could be lowered by a factor of 15 to $\left|\lambda_{132} \lambda_{231}^{*}\right| \leq 3 \times 10^{-4}$ for assumed superpartner masses of order $100 \mathrm{GeV} / c^{2}$

TABLE I. Summary of three data-taking series. The number of produced muonium atoms $N_{\mathrm{M}}$ and the upper limits on the conversion probability $p_{\mathrm{MM}}$ in $B_{0}=0.1 \mathrm{~T}$ magnetic field are given. The probability in zero field can be obtained through division by the interactiondependent reduction factor $S_{B}\left(B_{0}\right)$ of Table II. $S_{\mathrm{vol}}$ accounts for the finite fiducial volume.

\begin{tabular}{|c|c|c|c|c|c|}
\hline Measurement & $\begin{array}{l}\text { Duration of } \\
\text { data taking }\end{array}$ & $\begin{array}{c}N_{\mathrm{M}} \\
\text { (in vacuo) }\end{array}$ & $\begin{array}{c}\overline{\mathrm{M}} \text {-like events } \\
\text { (expected background) }\end{array}$ & $S_{\mathrm{vol}}$ & $\begin{array}{l}P_{\mathrm{MM}}(0.1 \mathrm{~T}) \\
(90 \% \text { C.L. })\end{array}$ \\
\hline 1 & $210 \mathrm{~h}$ & $1.4(1) \times 10^{9}$ & 0 [no] & 0.76 & $\leq 2.8 \times 10^{-9}$ \\
\hline 2 & $230 \mathrm{~h}$ & $2.4(2) \times 10^{9}$ & $0[\mathrm{no}]$ & 0.76 & $\leq 1.6 \times 10^{-9}$ \\
\hline 3 & $1290 \mathrm{~h}$ & $5.2(1) \times 10^{10}$ & $1[1.7(2)]$ & 0.82 & $\leq 9.0 \times 10^{-11}$ \\
\hline All & $1730 \mathrm{~h}$ & $5.6(2) \times 10^{10}$ & $1[1.7(2)]$ & 0.82 & $\leq 8.3 \times 10^{-11}$ \\
\hline
\end{tabular}



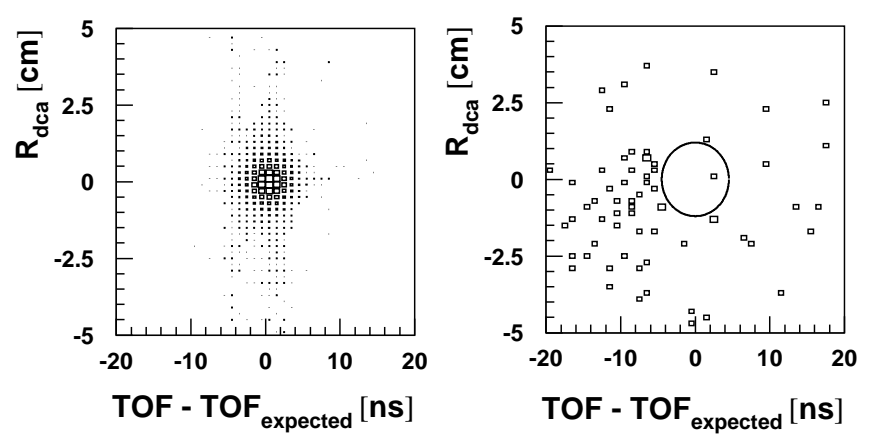

FIG. 4. The distribution of the distance of closest approach $\left(R_{\text {dca }}\right)$ between a track from an energetic particle in the magnetic spectrometer and the backprojection of the position on the MCP detector versus the TOF of the atomic shell particle for a muonium measurement (left) and for all data recorded in the third data-taking period of $1290 \mathrm{~h}$ while searching for antimuonium (right). One single event falls within a three-standard-deviation region of the expected TOF and $R_{\text {dca }}$ indicated by the ellipse. It is further characterized by $p_{T}=$ $16.2 \mathrm{MeV} / c$, two $\gamma$ 's at $E_{\gamma}=490$ and $560 \mathrm{keV}, t_{\mathrm{CsI}}=2 \mathrm{~ns}$, and a vertex $9.2 \mathrm{~mm}$ above the target. The events concentrated at low TOF and low $R_{\mathrm{dca}}$ correspond to the allowed decay $\mu \rightarrow 3 e+2 \nu$ and Bhabha scattering.

[25]. Furthermore, the achieved level of sensitivity allows one to narrow slightly the interval of allowed heavy muon neutrino masses in minimal left-right symmetry [4] (where a lower bound on $G_{\mathrm{MM}}$ exists, if muon neutrinos are heavier than $35 \mathrm{keV} / \mathrm{c}^{2}$ ) to $\approx 40 \mathrm{keV} / c^{2}$ up to the present experimental bound of $170 \mathrm{keV} / c^{2}[26]$.

In minimal left-right symmetric models, in which $\mathrm{MM}$ conversion is allowed, the process is intimately connected to the lepton family number-violating muon decay $\mu^{+} \rightarrow e^{+}+\nu_{\mu}+\bar{\nu}_{e}$. With the limit achieved in this experiment this decay is not an option for explaining the excess neutrino counts in the LSND neutrino experiment [27] at Los Alamos within these models [28], as the rate is too high.

The consequences for atomic physics of $\mathrm{M}$ are such that the expected level splitting in the ground state due to $\mathrm{M}-\overline{\mathrm{M}}$ interaction is below $1.5 \mathrm{~Hz} / \sqrt{S_{B}\left(B_{0}\right)}$ reassuring the validity of recent determinations of fundamental constants from atomic spectroscopy of the atom.

Further increased sensitivity to $M \bar{M}$ conversion could be expected, if the $\mathrm{M}$ production efficiency could be

TABLE II. Magnetic field correction factor $S_{B}\left(B_{0}\right)$ of muonium-antimuonium conversion probability for muonium atoms with statistically populated ground states [21,22].

\begin{tabular}{lccc}
\hline \hline & & & \\
$\quad$ Interaction type & $2.8 \mu \mathrm{T}$ & $0.1 \mathrm{~T}$ & $100 \mathrm{~T}$ \\
\hline$S S$ & 0.75 & 0.50 & 0.50 \\
$P P$ & 1.0 & 0.9 & 0.50 \\
$(V \pm A) \times(V \pm A)$ or & & & \\
$\quad(S \pm P) \times(S \pm P)$ & 0.75 & 0.35 & 0.0 \\
$(V \pm A) \times(V \mp A)$ or & & & \\
$(S \pm P) \times(S \mp P)$ & 0.95 & 0.78 & 0.67 \\
\hline \hline
\end{tabular}

enhanced, or from muon sources with significantly higher fluxes, e.g., at the front end of a future muon collider.

This work is supported in part by the German BMBF, the Swiss Nationalfond, the Russian FFR, and a NATO research grant. We thank P.H. Frampton, A. Halprin, P. Herczeg, W. S. Hou, R. N. Mohapatra, H.S. Pruys, and T. Sasaki for discussions on the implications of the measurements. The excellent support we received from PSI staff members was essential for successful extended running of the experiment.

[1] N. Cabbibo, Nuovo Cimento 19, 612 (1961).

[2] M. D. Cooper, in Intersections between Particle and $\mathrm{Nu}$ clear Physics, Sixth Conference, edited by T. W. Donnelly, AIP Conf. Proc. No. 412 (AIP, New York, 1998), p. 34.

[3] A. Halprin, Phys. Rev. Lett. 48, 1313 (1982).

[4] P. Herczeg and R. N. Mohapatra, Phys. Rev. Lett. 69, 2475 (1992).

[5] G. G. Wong and W. S. Hou, Phys. Rev. D 50, R2962 (1994); W. S. Hou and G. G. Wong, Phys. Rev. D 53, 1537 (1996).

[6] R. N. Mohapatra, Z. Phys. C 56, 117 (1992).

[7] A. Halprin and A. Masiero, Phys. Rev. D 48, 2987 (1993).

[8] H. Fujii et al., Phys. Rev. D 49, 559 (1994).

[9] P. H. Frampton, Phys. Rev. Lett. 69, 2889 (1992); see also hep-ph/9711281.

[10] D. L. Morgan, Jr., and V.W. Hughes, Phys. Rev. D 2, 1389 (1970); Phys. Rev. A 7, 1811 (1973).

[11] G. Feinberg and S. Weinberg, Phys. Rev. 123, 1439 (1961).

[12] B. E. Matthias et al., Phys. Rev. Lett. 66, 2716 (1991); for other techniques, see V.A. Gordeev et al., JETP Lett. 59, 589 (1994); T. M. Huber et al., Phys. Rev. D 41, 2709 (1990); B. Ni et al., Phys. Rev. D 48, 1976 (1993).

[13] R. Abela et al., Phys. Rev. Lett. 77, 1950 (1996).

[14] L. Michel, Proc. Phys. Soc. A 63, 514 (1950).

[15] L. Chatterjee et al., Phys. Rev. D 46, 5200 (1992).

[16] R. Abela et al., Z. Phys. C 56, S240 (1992).

[17] K. Woodle et al., Z. Phys. D 9, 59 (1988); see also A. C. Janissen et al., Phys. Rev. A 42, 161 (1990); G. A. Beer et al., Phys. Rev. Lett. 57, 671 (1986); R. F. Kiefl et al., Phys. Rev. B 26, 2432 (1982); G. M. Marshall et al., Phys. Lett. 65A, 351 (1978).

[18] P. V. Schmidt et al., Nucl. Instrum. Methods Phys. Res., Sect. A 376, 139 (1996).

[19] R. D. Cousin and V. L. Highland, Nucl. Instrum. Methods Phys. Res., Sect. A 320, 331 (1992).

[20] G. G. Wong and W. S. Hou, Phys. Lett. B 357, 145 (1995).

[21] K. Horikawa and K. Sasaki, Phys. Rev. D 53, 560 (1996).

[22] F. Cuypers and S. Davidson, Eur. Phys. J. C 2503 (1998).

[23] P. H. Frampton and M. Harada, hep-ph/9711448.

[24] P. H. Frampton (private communication).

[25] R. N. Mohapatra (private communication).

[26] K. Assamagan et al., Phys. Rev. D 53, 6065 (1996).

[27] C. Athanassopoulos et al., Phys. Rev. C 54, 2685 (1996); Phys. Rev. Lett. 81, 1774 (1998).

[28] P. Herczeg, in Beyond the Desert 1997, edited by H. V. Klapdor-Kleingrothaus and H. Päs (Institute of Physics Publishing, Bristol, 1998), p. 124. 